\title{
EMPIEMA PLEURICO CAUSATO DA \\ BLASTO-SCHIZOMYCES CAPITATUS IN \\ PAZIENTE CON SINDROME DI MOEBIUS
}

Fazii P.', Pelatti A.' ', Lococo A. ${ }^{2}$, Russi C.', Pistola F.', Stella

M.'; Crescenzi C. ';Clerico L.'; Cosentino L.'; Casaccia M.' ${ }^{2}$, Polilli E.' , Riario Sforza G. '.

' U.O.di Laboratorio Analisi Chimico-cliniche e Microbiologia ,

2 U.O. di Chirurgia Toracica P.O. "Spirito Santo",

Via Fonte Romana 8, 65 I 24 Pescara

La sindrome di Moebius è una rara patologia congenita la cui caratteristica principale è la paralisi facciale a causa della mancata o ridotta formazione dei nervi cranici VI e VII. Talora sono associati anche problemi agli altri nervi cranici e malformazioni varie. Descriviamo un caso di empiema pleurico causato da Blastoschizomyces capitatus in un soggetto abruzzese di 29 anni, affetto da sindrome di Moebius con ritardo psico-motorio. A causa di una insufficienza respiratoria acuta causata da una brocopolmonite bilaterale $a b$ ingestis, viene ricoverato presso il nostro nosocomio nel giugno 2004. La TAC del torace documenta la presenza di un idropneumotorace massivo sinistro iperteso con versamento parenchimale del lobo inferiore destro di tipo flogistico. Il paziente presenta una lieve anemia con leucocitosi neutrofila $(\mathrm{WBC}=15.300$, neutrofili $=84.1 \%)$. Vengono eseguite le cure del caso sia farmacologiche che chirurgiche con drenaggio toracico. Dal materiale purulento prelevato l'esame colturale evidenzia lo sviluppo di colonie di lievito. Il ceppo viene identificato mediante sistema API bioMerieux come appartenente alla specie $B$. capitatus. Su nostra indicazione vengono eseguiti altri esami microbiologici sia a livello del materiale raccolto dal drenaggio che dal cavo orale e dalle feci: in tutti viene evidenziata la presenza di B. capitatus. L'antimicogramma eseguito mediante sistema Sensititre consiglia l'uso del fluconazolo che viene prontamente immesso in terapia. Le radiografie eseguite successivamente documentano una soddisfacente riespansione parenchimale polmonare a sinistra. Alla fine del mese il paziente, apiretico e con un emocromo che testimonia la presenza di una lieve anemia ma, senza leucocitosi, viene dimesso.

Descriviamo il caso per la rarità delle segnalazioni di infezioni sostenute da $B$. capitatus, solitamente associate a stati di gravi immunodeficit mentre il nostro paziente era defedato ma non immunodeficiente. Questo da noi presentato sembra essere il primo caso descritto di empiema pleurico causato da B. capitatus. 